\title{
Rastreamento cognitivo em idosos esquizofrênicos institucionalizados
}

\section{Cognitive screening of institutionalized schizophrenic elderly patients}

Jerson Laks ${ }^{\mathrm{a}}$, Úrsula Vega ${ }^{\mathrm{b}}$, Cláudia Silberman $^{\mathrm{b}}$, Márcia Rozenthal $^{\mathrm{b}}$, Fortuneé N Nigri ${ }^{\mathrm{b}}$, Regina C Freitas ${ }^{\mathrm{b}}$, Mayze Machado $^{\mathrm{b}}$ e Eliasz Engelhardt ${ }^{\mathrm{c}}$

${ }^{a}$ Centro Para Pessoas com Doença de Alzheimer e Desordens Relacionadas (CDA/IPUB/UFRJ), Rio de Janeiro, RJ, Brasil. Faculdade de Ciências Médicas da Universidade Estadual do Rio de Janeiro, Rio de Janeiro, RJ, Brasil. ' Instituto Municipal de Assistência à Saúde Juliano Moreira, Rio de Janeiro, RJ, Brasil. 'Setor de Neurologia Cognitiva e do Comportamento do Instituto de Neurologia Deolindo Couto (INDC) da Universidade Federal do Rio de Janeiro, Rio de Janeiro, RJ, Brasil

Resumo Objetivos: Avaliar a cognição de pacientes esquizofrênicas idosas institucionalizadas. Comparar os subgrupos, divididos de acordo com o grau de escolaridade, quanto aos escores cognitivos e à duração da doença.

Métodos: Aplicou-se o Mini-Exame do Estado Mental (Mini-Mental State Examination - MEEM) e questionários de avaliação de duração do transtorno e de instrução e avaliou-se os medicamentos usados por classe e esquema posológico em 38 pacientes idosas institucionalizadas com diagnóstico de esquizofrenia segundo os critérios da DSM-IV. Os dados foram obtidos dos prontuários das pacientes e em entrevista direta com as mesmas.

O MEEM foi aplicado sem que os examinadores tivessem conhecimento do diagnóstico dos sujeitos, já que todas as 38 pacientes foram inicialmente avaliadas e somente depois disso os procedimentos para o diagnóstico foram levados adiante.

A média e o desvio-padrão foram obtidos para o grupo total e a significância determinada com o teste $\mathrm{t}(0,05)$.

Resultados: A média de idade desses indivíduos $(\mathrm{n}=38)$ foi de 69,42 $\pm 6,8(\min =60, \max =82)$ anos e a duração do transtorno foi de 40,36 $\pm 5,89$ ( $\min =29$, $\max =56$ ) anos. As pacientes analfabetas somam 18 , as que têm até 4 anos de educação são 14 e 6 têm mais de 4 e menos de 8 anos de educação.

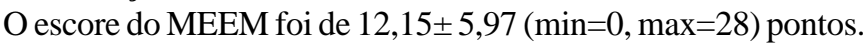

As pacientes com até 8 anos de educação formal $(n=20)$ tiveram MEEM de 14,05 $\pm 5,97$, idade de 70,15 $\pm 5,79$ anos e a duração do transtorno de 40,36 $\pm 6,44$. Já as pacientes analfabetas $(n=18)$, com idade de 68,61 \pm 7,56 anos, tiveram desempenho de MEEM 10,05 \pm 5,22, com 40,35 \pm 5,20 anos de doença.

Comparando-se os grupos de escolaridade baixa/média e de analfabetos quanto à idade, ao tempo de doença e ao escore do MEEM (14,05 \pm 5,97 e 10,05 \pm 5,22, respectivamente), somente este último item apresentou diferença estatisticamente significativa $(\mathrm{p}<0,05)$.

Conclusões: As pacientes esquizofrênicas idosas institucionalizadas no Instituto Municipal de Assistência à Saúde Juliano Moreira têm desempenho cognitivo medido pelo MEEM melhor do que os resultados de instituições americanas e inglesas.

O número de anos de estudo correlaciona-se positivamente com o desempenho, isto é, analfabetas têm desempenho significativamente pior que pacientes com educação baixa/média.

Apesar da longa duração, a pontuação no MEEM demonstra comprometimento cognitivo moderado, o que pode significar que haja também possibilidades adaptativas, desde que sob estrita supervisão.

Descritores Idoso. Esquizofrenia. Cognição.

Abstract Objective: To evaluate the cognition level of institutionalized schizophrenic elderly patients and compare groups according to years of education, disease duration and institutionalization.

Methods: The Mini-Mental State Examination (MMSE) and questionnaires for the evaluation of the disease duration and level of education were carried out in the 38 female institutionalized elderly patients with a diagnosis of schizophrenia according to DSM-IV criteria. Their medication use was assessed according to specific drug class and posology. Data was obtained from the patients' records and interview. 
The MMSE interviewers weren't aware of the patients' diagnoses, since all 38 patients were initially evaluated and only then the diagnostic procedures were performed.

The mean and standard deviation were obtained for the whole group and their level of significance was determined by the t-test $(0,05)$.

Results: Mean age of subjects $(n=38)$ was $69.42 \pm 6.8$ years old $(\min =60, \max =82)$ and the mean disease duration was $40.36 \pm 5.89$ years $(\min =29$, max-56). 18 patients were illiterate, 14 had 4 years of schooling and 6 had $4-8$ years of education.

The mean MMSE score was $12.15 \pm 5.97$ ( $\min =0, \max =28)$.

Patients with more than 8 years of education $(n=20)$ achieved a mean MMSE of 14.05 $\pm 5.97($ age $=70.15 \pm 5.79$ years old and disease duration $=40.36 \pm 6.44$ years $)$, and illiterate patients $(n=18$, age $=68.61 \pm 7.56$, disease duration $=40.35 \pm 5.20$ years) achieved a MMSE score of 10.05 \pm 5.22 .

Comparing MMSE scores of groups according to years of formal education, age, and disease duration the only statistically significant difference was observed in the number of years of education $(\mathrm{p}<0.05)$.

Conclusion: Elderly institutionalized schizophrenic patients had higher MMSE scores and cognitive performance than those living in institutions in the U.S. and United Kingdom as reported by the literature. Years of formal education have a positive influence on cognitive performance, ie., illiterate people have a poorer performance than patients who had low-to-intermediate level of education. Despite the long duration of the disorder, MMSE scores show moderate degree of impairment. These data may help to develop adaptive care for this population.

\section{Keywords Elderly. Schizophrenia. Cognition.}

\section{Introdução}

O comprometimento cognitivo é comum na esquizofrenia, ${ }^{1,2}$ afeta vários domínios neuropsicológicos e está mais associado à evolução de sintomas negativos. ${ }^{3}$ A determinação do estado cognitivo tem importância para se avaliar a capacidade de reabilitação e de vida em comunidade de populações com uma doença crônica e incapacitante como a esquizofrenia, em especial nos indivíduos idosos institucionalizados há longo tempo. É também um preditor mais consistente de má evolução do quadro psicótico do que os sintomas negativos quanto à capacidade de reabilitação e de adaptação ao meio por parte desses pacientes. Há uma correlação positiva entre o comprometimento cognitivo e o comprometimento adaptativo em pacientes esquizofrênicos idosos institucionalizados. ${ }^{2}$ Assim, a avaliação cognitiva desses pacientes pode trazer informações úteis para o desenvolvimento de estratégias de cuidado e proteção nesses casos.

Argumenta-se que o longo tempo de institucionalização pode ser o causador dos déficits cognitivos nesses pacientes devido ao ambiente pouco estimulante, ao excesso de medicação e a outros fatores que são encontrados nesses locais. ${ }^{2}$ No entanto há uma surpreendente estabilidade no comprometimento cognitivo de pacientes esquizofrênicos nas várias culturas e diferentes ambientes de tratamento, tornando esses dados generalizáveis para todos os pacientes e não apenas para os institucionalizados. $^{4}$

Em esquizofrênicos idosos, surge a questão do diagnóstico diferencial com quadros demenciais que possam se superpor ao estado de doença psiquiátrica primária. $\mathrm{O}$ conhecimento do perfil cognitivo desses pacientes pode eventualmente direcionar os serviços de assistência e reabilitação no mesmo sentido daqueles oferecidos para pacientes com demências degenerativas. Embora vários estudos tenham se ocupado de avaliações cognitivas extensas, complexas e longas para a determinação de perfis cognitivos em pequenas amostras de pacientes esquizofrênicos que aceitam cooperar, ${ }^{4}$ o rastreamento de déficits cognitivos de amostras mais numerosas de pacientes deve ser obtida através de aplicação de um teste mais rápido e que gere respostas interpretáveis à luz da sua normatização em populações normais, naqueles que tem quadros "orgânicos" e nas normatizações obtidas com pacientes com esquizofrenia. O Mini-Exame do Estado Mental (Mini-Mental State Examination, MEEM) $)^{5}$ preenche todos esses critérios acima relacionados e, embora tenha especificidade limitada para síndromes clínicas individuais, ${ }^{6}$ já foi utilizado em mais de 30 estudos publicados sobre esquizofrenia. ${ }^{4}$ De fácil aplicação, o MEEM ${ }^{5}$ é um teste semi-estruturado com pontuação máxima de 30, sendo seu ponto de corte para comprometimento cognitivo situado entre 23 e 24 pontos. A escolaridade e a idade têm efeito sobre o desempenho no teste, sendo que a pontuação diminui com a idade e aumenta com a educação, como observado em estudos de normatização em populações idosas não dementes, na comunidade e em instituições, e em populações sem doença neuropsiquiátrica. ${ }^{6-10}$ Nos estudos normativos em amostras de idosos na comunidade, os valores de referência do $\mathrm{MEEM}^{5}$ específicos para idade e educação foram para a faixa entre 0 e 4 anos de educação, de 25,7 $\pm 3,4$ para pessoas entre 65 e 69 anos e, de 25,7 $\pm 2,7$ para as idades entre 70 e 74 anos, 25,4 $\pm 1,9$ para as idades entre 75 e 79 anos e $24,5 \pm 2,8$ para a faixa etária acima de 85 anos. Para 5 a 8 anos de educação, os resultados obtidos foram os seguintes: $26,9 \pm 2,8$ (65 a 69 anos), 27,0 $\pm 2,5$ (70 a 74 anos), 26,4 $\pm 2,0$ (75 a 79 anos), 25,8 $\pm 2,0$ (80 a 84) e 25,2 $\pm 1,8$ (acima de 85 anos). A tabela normativa abrange todas as faixas educacionais e ainda posiciona os escores em percentis de $5 \%$, $25 \%$ e $50 \%$ para cada faixa etária e educacional. ${ }^{9}$ Os escores obtidos nesse último estudo são surpreendentemente altos, 
podendo corresponder a um fenômeno cultural e epidemiológico específico canadense. A própria comparação entre os grupos culturais e de línguas diferentes (inglês e francês) gerou diferenças estatisticamente significativas. Outro grande estudo de normatização na população de 18 até mais de 85 anos de idade gerou escore do MEEM $^{5}$ de $23 \pm 1,9$ pontos em pessoas com 0 a 4 anos de educação ( 60 a 64 anos), $22 \pm 1,9$ (65 a 69 anos), $22 \pm 1,7$ ( 70 a 74 anos), $21 \pm 2,0$ ( 75 a 79 anos), $20 \pm 2,2$ (80 a 84 anos) e $19 \pm 2,9$ (para acima de 85 anos). A faixa educacional de 5 a 8 anos de escolaridade teve pontuação marcadamente maior, com 26 pontos dos 60 aos 65 anos e até 23 pontos para acima de 85 anos. $^{6}$ A avaliação de 130 idosos de 65 a 98 anos sadios na comunidade com atividades de vida diária normais no Rio de Janeiro deu como resultado do MEEM ${ }^{5}$ 26,54 $\pm 2,55$ pontos para todas as faixas educacionais. Os indivíduos de escolaridade baixa $(n=32)$ obtiveram $24,53 \pm 3,07$ pontos e os de média escolaridade conseguiram $26,38 \pm 2,15$ pontos. Nesse estudo houve apenas 3 analfabetos, não sendo então possível estabelecer dados normativos para comparação. ${ }^{8}$

Num estudo recente de Leon et al (1998), ${ }^{4}$ foi realizada uma análise fatorial do $\mathrm{MEEM}^{5} \mathrm{em} 80$ pacientes esquizofrênicos institucionalizados com média de idade de 38 anos, 11 anos de educação formal, primeiro episódio aos 19 anos e 6 anos de tempo de hospitalização. Essa análise foi feita com os itens do MEEM $^{5}$ divididos em três grupos: uma subescala frontal incluindo os subtestes 7 seriados, soletrar "mundo" ao contrário, desenho e orientação temporal, uma subescala de memória formada por registro, evocação das três palavras e repetição da frase, enquanto a subescala espacial foi constituída de escrita e orientação espacial. ${ }^{4}$ Nos pacientes estudados, os escores variaram de acordo com a idade e a escolaridade, como em todos os demais estudos sobre o assunto, embora não tenha ocorrido a generalização do comprometimento à medida que progride a duração do transtorno como acontece com os processos demenciais degenerativos. Essa seria uma característica da esquizofrenia a qual o MEEM $^{5}$ seria sensível quando dividido nos três agrupamentos acima delineados. Esquizofrênicos raramente erram os itens de registro, repetição, comando, denominação e leitura. ${ }^{4} \mathrm{Já}$ a subescala frontal tem inclusive correlação positiva com o teste de trilhas $\mathrm{A} / \mathrm{B}$, o que reflete $\mathrm{o}$ acometimento de função frontal nessa desordem.

No presente estudo, foram avaliadas as pacientes esquizofrênicas do Instituto Municipal de Assistência à Saúde Juliano Moreira (IMASJM) com rastreamento cognitivo utilizando o MEEM. ${ }^{5}$ As pacientes foram divididas em grupos de acordo com o grau de escolaridade; esses grupos foram comparados entre si com relação aos escores no $\mathrm{MEEM}^{5}$ e quanto ao tempo de duração da doença.

\section{Métodos}

O IMASJM está situado na zona Oeste do Rio de Janeiro e conta com cerca de 900 internos. Foram entrevistadas todas as pacientes de um núcleo feminino, a Unidade Hospitalar Franco da Rocha $(n=65)$, visando fazer o diagnóstico de esquizofrenia de acordo com critérios da DSM IV. ${ }^{11}$ Aplicou-se o MEEM ${ }^{5}$ e questionário para avaliar a duração da doença e o número de anos de medicamentos utilizados por classe química e esquema posológico. Os dados foram obtidos dos prontuários das pacientes e em entrevista direta com as mesmas.

$\mathrm{O}_{\mathrm{MEEM}}{ }^{5}$ foi aplicado sem que os examinadores tivessem conhecimento do diagnóstico dos sujeitos, já que todas as 65 pacientes foram inicialmente avaliadas e somente depois disso os procedimentos para o diagnóstico foram levados adiante.

As médias e os desvios-padrão foram obtidos para o grupo total e para as faixas educacionais e essas variáveis foram comparadas entre si através do teste paramétrico t de Student. O nível de significância adotado foi de 0,05.

\section{Resultados}

A Tabela 1 mostra os resultados dos grupos divididos por tempo de escolaridade. A média do $\mathrm{MEEM}^{5}$ foi de $12,15 \pm 5,97$ $(\min =0, \max =28)$ pontos.

Do total de pacientes, não foi possível colher informação da duração da doença em uma pessoa.

Comparando-se o grupo de escolaridade baixa/média com o de analfabetas em relação à idade $(\mathrm{t}=0,6823 ; \mathrm{p}=0,4)$, duração da doença $(t=0,007646 ; p=0,99)$ e escore do MEEM, somente esse último item apresentou diferença estatisticamente significativa $(\mathrm{t}=2,124 ; \mathrm{p}<0,05)$.

A Tabela 2 mostra os medicamentos usados pelas pacientes. Os seguintes medicamentos também constam das prescrições: levomepromazina $25 \mathrm{mg}(\mathrm{n}=1)$, clonazepam $4 \mathrm{mg}(\mathrm{n}=1)$, risperidona $6 \mathrm{mg}(\mathrm{n}=1)$, biperideno $2 \mathrm{mg}$ e $4 \mathrm{mg}(\mathrm{n}=2)$, imipramina $50 \mathrm{mg}(\mathrm{n}=1)$, fenitoína $100 \mathrm{mg}(\mathrm{n}=1)$, carbamazepina $600 \mathrm{mg}(\mathrm{n}=2)$ e fenobarbital $100 \mathrm{mg}(\mathrm{n}=1)$.

A distribuição de uso de medicamentos por grupos de escolaridade não apresentou diferenças que pudessem ser estatisticamente analisadas. As doses de haloperidol usadas pelas pacientes analfabetas e pelo grupo de escolaridade baixa/média foi de 5,0 mg $\pm 3,05 \mathrm{mg}$ e 4,0 $\mathrm{mg} \pm 4,29 \mathrm{mg}$, respectivamente $(t=0,6078 ; p=0,55$. Teste $t$ não pareado com correção de Welch). Ambos os grupos tiveram cinco pacientes sem uso de medicação e o mesmo número de sujeitos usou prometazina com doses equivalentes.

\section{Discussão}

O grupo de baixa/média escolaridade ( 0 a 8 anos de estudo formal) teve desempenho significativamente mais alto do que o grupo de analfabetos $(\mathrm{p}<0,05)$, com 14 pontos e 10 pontos, respectivamente. As demais variáveis estudadas, duração

Tabela 1 - Idade, anos de doença e escores do MEEM dos grupos por escolaridade ${ }^{\star}$

\begin{tabular}{lcc}
\hline & Analfabetas $(\mathrm{n}=18)$ & Até 8 anos $(\mathrm{n}=20)$ \\
\hline Idade & $68,61 \pm 7,56$ & $70,15 \pm 5,79$ \\
Anos de doença & $40,35 \pm 5,20$ & $40,36 \pm 6,44$ \\
MMSE & $10,05 \pm 5,22$ & $14,05 \pm 5,97$ \\
\hline
\end{tabular}

*dados expressos por média e desvio-padrão

Tabela 2 - Medicamentos utilizados pelas pacientes*

\begin{tabular}{lcc}
\hline Medicamento & $\mathrm{n}$ & $\mathrm{mg} / \mathrm{dia}$ \\
\hline Haloperidol & 21 & $4,38 \pm 3,30$ \\
Clorpromazina & 3 & $116,66 \pm 62,36$ \\
Diazepam & 4 & $7,50 \pm 2,50$ \\
Prometazina & 16 & $32,81 \pm 11,58$ \\
\hline${ }^{*}$ resultados expressos em média e desvio-padrão. &
\end{tabular}


da doença, idade e duração da institucionalização, não apresentaram diferenças entre os dois grupos. Assim, podemos afirmar que, nesta amostra de pacientes esquizofrênicas crônicas, o número de anos de educação formal está associado positivamente no desempenho cognitivo de modo marcante, da mesma maneira já observada em vários outros trabalhos sobre o assunto. $2,6,8,9,12$

As pacientes analfabetas tiveram escore médio de 10 pontos. Ao se utilizar o ponto de corte $13^{12}$ para este grupo observa-se que há disparidade entre duração do transtorno, tempo de institucionalização e, o que seria de se esperar, de comprometimento cognitivo. O resultado total médio do $\mathrm{MEEM}^{5}$ da amostra situou-se muito próximo do ponto de corte para comprometimento cognitivo (12 pontos), enquanto o $\mathrm{MEEM}^{5}$ para a faixa baixa/ média de escolaridade ficou acima deste ponto de corte (14 pontos). Somente as analfabetas ficaram abaixo (10 pontos). No entanto, após o longo tempo de institucionalização e de doença, o comprometimento cognitivo medido pelo MEEM $^{5}$ não parece tão afetado. É de se notar ainda que uma das pacientes obteve 28 pontos no MEEM, ${ }^{5}$ normal mesmo para pessoas sadias.

Os dados deste estudo são diferentes dos obtidos por Harvey et $\mathrm{al}^{2}$ em Londres e em Nova York, em grupo de educação baixa/ média. Enquanto o escore do grupo no IMASJM foi de 14 pontos, os americanos e ingleses tiveram 10 pontos. No entanto, os pacientes americanos e ingleses eram mais velhos que as brasileiras (81 vs 70 anos) e tinham educação formal de 9 anos em média. É possível que a idade tenha exercido seu efeito negativo sobre o desempenho no MEEM $^{5}$ dos grupos inglês e americano em comparação ao grupo deste trabalho.

A avaliação da correlação entre o desempenho no $\mathrm{MEEM}^{5}$ e a presença de discinesia tardia (DT) em pacientes esquizofrênicos idosos institucionalizados mostrou resultados do MEEM $^{5}$ de $13,32 \pm 10,58$ e 10,67 $\pm 9,80$ para indivíduos sem DT e com DT, respectivamente. ${ }^{13}$ Embora não houvesse diferença significativa entre os dois grupos, resultados estatisticamente significativos apareceram quando avaliou-se somente a presença de itens de DT nas regiões oral e facial. A amostra desse trabalho contou com 121 pessoas (53 mulheres) com média de 74 anos de idade, 9 anos de educação formal e 49 anos de duração do transtorno e doses médias de neurolépticos bem maiores do que as usadas pelos pacientes do IMASJM. Embora não tenhamos avaliado as pacientes quanto à presença de DT, pode-se observar que os resultados do presente trabalho concordam com aqueles dos pacientes sem DT, embora o nível de escolaridade e a média de idade sejam maiores no estudo de Byne et al. ${ }^{13}$

\section{Referências}

1. Braff DL, Heaton R, Kuck J, Cullum M, Moranville J, Grant I, et al. The generalized pattern of neuropsychological deficits in outpatients with chronic schizophrenia with heterogeneous Wisconsin Card Sorting Test results. Arch Gen Psychiatry 1991;48:891-8

2. Harvey PD, Leff J, Trieman N, Anderson J, Davidson M. Cognitive impairment in geriatric chronic schizophrenic patients: a crosssectional study in New York and London. Int J Geriat Psychiatry 1997;12:1001-7.
Algumas questões devem ser levadas em consideração para a avaliação dos dados aqui obtidos. A amostra de pacientes foi exclusivamente do sexo feminino, o que pode contribuir para um viés da amostra, já que pacientes esquizofrênicos masculinos poderiam ter maior gravidade de doença clínica e gerar diferenças nos escores totais do MEEM. ${ }^{5}$ No entanto, nenhum dos estudos referenciados apresenta qualquer comparação entre sexos, limitando-se a relatar o número de pessoas estudadas do sexo masculino e feminino. Não fica claro, assim, se o sexo nesses estudos é uma variável que determina diferenças significativas. Não foram apresentados dados sobre correlações com uso dos medicamentos, discinesia tardia e comprometimento cognitivo, uma vez que os números não foram representativos o suficiente para permitir discussão sobre o assunto. De qualquer forma, o estudo está sendo levado adiante agora com uma amostra de pacientes do sexo masculino, o que também acarretará aumento da amostra total, possibilitando um tratamento estatístico mais refinado e conclusões mais firmes.

O número de pessoas com mais de 8 anos de escolaridade $(n=6)$ não permitiu fazer qualquer comparação entre as faixas baixa e média separadamente. Essa é uma diferença marcante entre a casuística brasileira e as americanas e inglesas, nas quais o tempo médio de escolaridade é de 9 anos. Embora os dados brasileiros devam ser comparados com cautela com os obtidos nesses estudos, é interessante notar que o grupo de analfabetos teve desempenho significativamente pior do que todos os grupos com educação formal baixa e média. Se o número de anos de escolaridade formal influencia na reserva cerebral, a ausência de escolaridade em pacientes que são afetados por uma doença tão grave e incapacitante como a esquizofrenia deve ser estudada à luz de suas conseqüências para a integração na vida social e de atividades de vida diária.

\section{Conclusão}

As pacientes esquizofrênicas idosas institucionalizadas no IMASJM têm desempenho cognitivo medido pelo MEEM ${ }^{5}$ melhor do que aqueles apontados por instituições americanas e inglesas. ${ }^{2}$

O número de anos de educação formal influencia positivamente o desempenho, isto é, pacientes analfabetas têm desempenho significativamente pior que pacientes com educação baixa/média. Esses dados devem ser levados em consideração no planejamento de desinstitucionalização e/ou programas de proteção para esses pacientes, já que as capacidades adaptativas dependem do perfil cognitivo.
3. Breier A, Schreiber JL, Dyer J, Pickar D. National Institute of Mental Health Longitudinal study of schizophrenia: Prognosis and predictors of outcome. Arch Gen Psychiat 1991;48:239-46.

4. de Leon J, Baca-García E, Simpson GM. A factor analysis of the Mini-Mental State Examination in schizophrenic disordes. Acta Psychiatr Scand 1998;98:366-8.

5. Folstein MF, Folstein SE, McHugh PR. "Mini-Mental State": a practical method for grading the cognitive state of patients for the clinician. J Psychiatr Res 1975;12:189-98. 
6. Crum RM, Anthony JC, Basset SS, Folstein MF. Population-based norms for the Mini-Mental State Examination by age and educational level. JAMA 1993;269(18):2386-91.

7. Engelhardt E, Laks J, Rozenthal M, von Poser NAS, Menkes C, Franco Neto CPB, et al. Idosos velhos ("oldest old"): rastreamento cognitivo com o MMSE. Rev Bras Neurol 1997;33(4):201-6.

8. Engelhardt E, Laks J, Rozenthal M, Marinho VM, Silberman C, Balassiano B, et al. MMSE em idosos normais e o binômio idade/ escolaridade. Pôster no XVII Congresso Brasileiro de Psiquiatria; 1998 Outubro; Fortaleza.

9. Bravo G, Hérbert R. Age and education-specific reference values for the Mini-Mental State Examinations derived from a non-demented elderly population. Int J Geriat Psychiatry 1997;12:1008-18.

10. Cummings JL. Mini-Mental State Examination. Norms, normals, and numbers. JAMA 1993;269(18):2420-1.

11. American Psychiatric Association. Diagnostic and statistical manual of mental disorders. $4^{\text {th }}$ edition. Washington (DC): American Psychiatric Press; 1994.
12. Bertolucci PHF, Brucki SMD, Campacci SR, Juliano Y. O mini-exame do estado mental. Impacto da escolaridade. Arq Neuropsiquiatr 1994;52(1):1-7.

13. Byne W, White L, Parella M, Adams R, Harvey PD, Davis KL. Tardive dyskinesia in a chronically institutionalized population of elderly schizophrenic patients: prevalence and association with cognitive impairment. Int J Geriat Psychiatry 1998;13:473-9.

\section{Correspondência}

Jerson Laks

Av. Nossa Senhora de Copacabana, 749/802

22050-000 Rio de Janeiro, RJ, Brasil

E-mail:jersonlk@iis.com.br 